\title{
Infección congénita por citomegalovirus en recién nacidos del estado de San Luis Potosí, México
}

Daniel E Noyola, M en C, (1) Lorena Matienzo-Serment, MC, (1) Sergio O Rodríguez-Vidal, QFB, (2) Uciel R Ochoa-Pérez, MC, ${ }^{(1,2)}$ Juan M Piña-Granja, QFB, ${ }^{(2)}$ Christian A García-Sepúlveda, D en C. ${ }^{(3)}$

\begin{abstract}
Noyola DE, Matienzo-Serment L, Rodríguez-Vidal SO, Ochoa-Pérez UR, Piña-Granja JM, García-Sepúlveda CA. Infección congénita por citomegalovirus en recién nacidos del estado de San Luis Potosí, México. Salud Publica Mex 20II;53:5 I3-5I5.
\end{abstract}

\section{Resumen}

Objetivo. Determinar la prevalencia de infección congénita por citomegalovirus en recién nacidos participantes en el programa de tamiz neonatal de los Servicios de Salud de San Luis Potosí. Material y métodos. Se evaluó la presencia de citomegalovirus en muestras de sangre almacenadas en papel filtro. Resultados. Se detectó la presencia de citomegalovirus en I0 $(0.68 \%)$ de I 457 muestras estudiadas. No se encontraron diferencias en las características de los recién nacidos con infección congénita en comparación con aquéllos sin infección. Conclusiones. Es necesario concientizar a los profesionales de la salud sobre la prevalencia e impacto de la infección congénita por citomegalovirus.

Palabras clave: citomegalovirus; infecciones por citomegalovirus; tamizaje neonatal; México
Noyola DE, Matienzo-Serment L, Rodríguez-Vidal SO, Ochoa-Pérez UR, Piña-Granja JM, García-Sepúlveda CA. Congenital cytomegalovirus infection in newborn infants from the state of San Luis Potosí, Mexico.

Salud Publica Mex 20I I;53:5I3-5I5.

\section{Abstract}

Objective. To determine the prevalence of congenital cytomegalovirus infection in newborn infants included in the neonatal screening program coordinated by the State Health Services in San Luis Potosí. Material and Methods. We evaluated the presence of cytomegalovirus in blood samples stored in filter paper. Results. Cytomegalovirus was detected in $10(0.68 \%)$ of the I 457 samples included in the study. There were no differences in the characteristics of infants with congenital infection compared to those without infection. Conclusions. It is necessary to increase awareness of health professionals regarding the prevalence and impact of congenital cytomegalovirus infection.

Key words:cytomegalovirus; cytomegalovirus infections; neonatal screening; Mexico
$\mathrm{C}^{1}$ citomegalovirus (CMV) es la principal causa de ¿infección congénita en el ámbito mundial. ${ }^{1,2}$ Como consecuencia de esta infección, miles de niños padecen de discapacidad permanente cada año. ${ }^{3}$ La información acerca del impacto de este virus como causa de infección congénita y la carga de enfermedad que supone para los sistemas de salud en Latinoamérica es escasa..$^{4-9}$ En México un estudio realizado en San Luis Potosí (SLP) confirmó la infección por CMV en $0.89 \%$ de los recién nacidos evaluados. ${ }^{9}$ Dicho estudio tiene la limitante de haberse realizado en un solo hospital. En el presente estudio determinamos la prevalencia de infección con-

(I) Departamento de Microbiología, Facultad de Medicina, Universidad Autónoma de San Luis Potosí. San Luis Potosí, México.

(2) Laboratorio Estatal de Salud Pública, Servicios de Salud del Estado de San Luis Potosí. San Luis Potosí, México.

(3) Laboratorio de Genómica Viral y Humana, Facultad de Medicina, Universidad Autónoma de San Luis Potosí. San Luis Potosí, México. 
génita por CMV en recién nacidos de unidades médicas de la Secretaría de Salud y de hospitales privados que participan en el programa de tamiz neonatal de los Servicios de Salud de SLP.

\section{Material y métodos}

Se utilizaron muestras de sangre seca conservadas en papel filtro, obtenidas por el programa de tamiz neonatal para hipotiroidismo congénito en el estado de SLP entre el 1 de junio de 2008 y el 31 de mayo de 2009. Éstas permanecieron almacenadas en el Laboratorio Estatal de Salud Pública del Estado de SLP a temperatura ambiente y periódicamente son desechadas. El protocolo de investigación fue aprobado por el Comité de Ética e Investigación de los Servicios de Salud del Estado de SLP.

Con el objetivo de determinar la prevalencia de infección por CMV se tomaron 1500 tarjetas de papel filtro al azar de las recabadas durante un año $(\mathrm{n}=26186)$ empleando un algoritmo computacional. Este número de tarjetas se derivó del cálculo efectuado considerando la prevalencia de infección congénita por CMV reportada a nivel mundial $(1 \%)$, la prevalencia mínima esperada $(0.5 \%)$, el número de tarjetas recabadas durante el periodo de estudio (26186) y un nivel de confianza de $95 \%$. La estimación original fue de 1438 muestras que se corrigió a 1500 considerando la posibilidad de pérdidas de acuerdo con criterios de exclusión establecidos para este estudio (recién nacidos de madres que no residen habitualmente en SLP y ausencia de muestra para la realización del estudio molecular).

La detección de CMV se realizó a través de un ensayo de PCR anidada empleando protocolos previamente descritos. ${ }^{10}$ La extracción de ADN se realizó manualmente empleando una técnica previamente establecida en nuestro laboratorio.

Se analizaron las características de los recién nacidos comparando entre aquellos con y sin infección congénita por CMV. Las comparaciones se realizaron utilizando la prueba exacta de Fisher (para variables categóricas) y $t$ de student (para variables continuas). El análisis estadístico se efectuó mediante el software SPSS para Windows (versión 14.0, SPSS Inc. Chicago, IL, EUA).

\section{Resultados}

De las 1500 tarjetas seleccionadas para el estudio, 43 fueron excluidas (12 en que no se contó con la tarjeta de papel filtro y 31 pertenecientes a hijos de madres cuya residencia no era el estado de SLP). Por lo tanto, la población final de estudio constó de 1457 muestras. No se observaron diferencias significativas en las características de los casos incluidos y excluidos del estudio. Se incluyeron en el estudio muestras de todas las regiones del estado. Se observó una elevada correlación entre el número de casos estudiados y el número de nacimientos registrados en cada municipio durante 2008 (coeficiente de correlación de $0.98 ; p<0.001$ ).

De los recién nacidos estudiados 725 (49.8\%) fueron del sexo femenino y $732(50.2 \%)$ del sexo masculino. El peso promedio al nacer fue de $3161.34 \mathrm{~g}$ y la talla promedio de $50.22 \mathrm{~cm}$. Se confirmó la presencia de CMV en 10 [0.68\%; intervalo de confianza del 95\% (IC), 0.35-1.22] de las muestras estudiadas. Las tarjetas positivas para $\mathrm{CMV}$ incluyeron a pacientes de las cuatro regiones geográficas del estado. Se observó una mayor prevalencia en la Zona del Altiplano (2.8\%; IC, 0.7-7.4) en comparación con la Zona Media (1.1\%; IC, 0.2-3.7), Zona Huasteca $(0.7 \%$; IC, $0.2-1.9)$ y Zona Centro $(0.3 \%$; IC, 0.04-0.9). Estas diferencias deben de ser consideradas con cautela, dado que el tamaño de la muestra no se calculó para estimar la prevalencia en cada una de las regiones. No se encontró ninguna asociación estadísticamente significativa entre las variables analizadas (cuadro I).

\section{Cuadro I \\ Características de recién nacidos con y sin infección congénita por citomegalovirus (CMV). San Luis Potosí, México, 2008-2009}

Caracteristica

Sexo femenino

Recién nacido de pretérmino

Malformación congénita mayor

Peso al nacimiento $(\mathrm{g})^{*}$

Talla al nacimiento $(\mathrm{cm})^{*}$

Edad materna (años)*

\footnotetext{
* Los datos representan media y desviación estándar
}

Sin infección por CMV $(n=1447)$

Con infección por CMV $(n=10)$

$720 / 1447(49.8 \%)$

$50 / 1432(3.5 \%)$

$13 / 1447(0.9 \%)$

$3160.54(491.59)$

$50.22 \quad(2.72)$

$24.81 \quad$ (6.39)

39)

(2)

$5 / 10(50 \%)$

$0 / 10(0 \%)$

$0 / 10(0 \%)$

3278 (636.4I)

$51 \quad(2.40)$

$23.3(6.91)$

0.37

0.46




\section{Discusión}

Este es el primer estudio en que se evalúa, con una muestra representativa de la demografía de todo un estado, la prevalencia de la infección congénita por CMV en nuestro país. Nuestro estudio presenta algunas limitaciones, incluyendo el carácter transversal y retrospectivo de la obtención de la muestra. Dado que la muestra se seleccionó a través del programa de tamiz neonatal de la Secretaría de Salud, es posible que algunos segmentos de la población no estén representados. Sin embargo, se estudiaron recién nacidos de todas las regiones del estado y encontramos una alta correlación entre el número de casos estudiados y el número de nacimientos registrados por municipio durante 2008.

Para este estudio hemos utilizado una técnica de biología molecular que permite la detección de este virus en muestras almacenadas, incluso durante años, a temperatura ambiente. ${ }^{11,12}$ Varios estudios han reportado que es posible alcanzar una sensibilidad diagnóstica cercana a $100 \%$ a partir de muestras de sangre seca en tarjetas de papel filtro. ${ }^{13,14}$ Sin embargo, Boppana y colaboradores recientemente reportaron que el uso de sangre preservada en papel filtro para la detección de CMV pudiera limitar la sensibilidad diagnóstica a solamente $28-34 \% .{ }^{15}$ Por lo tanto, la prevalencia encontrada en nuestra población $(0.68 \%)$ podría considerarse como una prevalencia mínima haciendo posible que el impacto de este virus sea incluso mayor al estimado. Aun así, nuestros resultados son de utilidad para estimar el número de casos de infección congénita que ocurren en nuestro estado o país. Tomando en cuenta la alta frecuencia de secuelas permanentes, aún en recién nacidos que presentan infección asintomática al nacimiento, el impacto de esta infección en nuestra población es considerable. ${ }^{4,16}$ Es necesario concientizar a la comunidad médica y sanitaria acerca de la seriedad de este problema de salud y su prevalencia, así como desarrollar métodos de diagnóstico más eficientes y estrategias preventivas que permitan reducir el impacto de esta infección en nuestra población.

\section{Agradecimientos}

Agradecemos la ayuda técnica de la QFB Alba Elizabeth Hernández Salinas y de la QFB Lidia Margarita Barrios Compeán, así como el apoyo de la QFB Virginia Juárez, directora del Laboratorio Estatal de Salud Pública del estado de San Luis Potosí para la realización de este proyecto. Este proyecto fue realizado con el apoyo del Fondo Sectorial de Investigación en Salud y Seguridad Social (Proyecto Clave CONACYT-SALUD-2009-01-115487).
Declaración de conflicto de intereses: Los autores declararon no tener conflicto de intereses.

\section{Referencias}

I. Demmler GJ. Congenital cytomegalovirus infection and disease. Adv Pediatr Infect Dis 1996; II:I35-162.

2. Kenneson A, Cannon MJ. Review and meta-analysis of the epidemiology of congenital cytomegalovirus (CMV) infection. Rev Med Virol 2007; 17:253-276

3. Jeon J, Victor M, Adler SP, Arwady A, Demmler G, Fowler K, et al. Knowledge and awareness of congenital cytomegalovirus among women. Infect Dis Obstet Gynecol 2006:80383.

4. Noyola DE, Jiménez-Capdeville ME, Demmler-Harrison GJ. Central nervous system disorders in infants with congenital cytomegalovirus infection. Neurol Res 2010; 32:278-284.

5. Mussi-Pinhata MM, Yamamoto AY, Moura-Brito RM, de Lima-Isaac M, de Carvalho e Oliveira PF, Boppana S, et al. Birth prevalence and natural history of congenital cytomegalovirus infection in a highly seroimmune population. Clin Infect Dis 2009; 49:522-528.

6. Yamamoto AY, Mussi-Pinhata MM, Cristina P, Pinto G, Moraes-Figueiredo LT, Jorge SM. Congenital cytomegalovirus infection in preterm and fullterm newborn infants from a population with a high seroprevalence rate. Pediatr Infect Dis J 200I; 20:188-192.

7. Gomila A, Rivas N, López EL. Infección congénita por citomegalovirus. An Pediatr (Barc) 2008; 69:311-315.

8. Stagno S, Dworsky ME, Torres J, Mesa T, Hirsh T. Prevalence and importance of congenital cytomegalovirus infection in three different populations. J Pediatr 1982; 101:897-900.

9. Noyola DE, Mejía-Elizondo AR, Canseco-Lima JM, Allende-Carrera R, Hernández-Salinas AE, Ramírez-Zacarías JL. Congenital cytomegalovirus infection in San Luis Potosi, Mexico. Pediatr Infect Dis J 2003; 22:89-90. 10. Monsiváis-Urenda A, Noyola-Cherpitel D, Hernández-Salinas A, García-Sepúlveda C, Romo N, Baranda L, et al. Influence of human cytomegalovirus infection on the NK cell receptor repertoire in children. Eur J Immunol 2010; 40:1418-1427.

II. Barbi M, Binda S, Caroppo S. Diagnosis of congenital CMV infection via dried blood spots. Rev Med Virol 2006;16:385-392.

12. Atkinson C, Walter S, Sharland M, Tookey P, Luck S, Peckham C, et al. Use of stored dried blood spots for retrospective diagnosis of congenital CMV. J Med Virol 2009; 8I:I394-I 398.

13. Barbi M, Binda S, Primache V, Caroppo S, Didò P, Guidotti P, et al. Cytomegalovirus DNA detection in Guthrie cards: a powerful tool for diagnosing congenital infection. J Clin Virol 2000; I7:159-165.

14. Binda S, Mammoliti A, Primache V, Didò P, Corbetta C, Mosca F, et al. Pp65 antigenemia, plasma real-time PCR and DBS test in symptomatic and asymptomatic cytomegalovirus congenitally infected newborns. BMC Infect Dis 2010;10:24.

I5. Boppana SB, Ross SA, Novak Z, Shimamura M, Tolan RW Jr, Palmer $A L$, et al. Dried blood spot real-time polymerase chain reaction assays to screen newborns for congenital cytomegalovirus infection. JAMA 2010; 303:1375-1382.

16. Baquero-Artigao F. Grupo de estudio de la infección congénita por citomegalovirus de la Sociedad Española de Infectología Pediátrica. Documento de consenso de la Sociedad Española de Infectología Pediátrica sobre el diagnóstico y el tratamiento de la infección congénita por citomegalovirus. An Pediatr (Barc) 2009; 71:535-547. 